\title{
EFFECT OF ELECTRIC AND MAGNETIC FIELDS ON DONOR STATES IN SEMICONDUCTOR QUANTUM WELLS
}

\author{
S. ROLNIK AND J. ADAMOWSKI \\ Faculty of Physics and Nuclear Techniques \\ Technical University of Mining and Metallurgy (AGH) \\ Al. Mickiewicza 30, 30-059 Kraków, Poland
}

\begin{abstract}
An influence of static external electric and magnetic fields on donor states in a quantum well is studied by variational means. The energies of the $1 s$ and $2 p_{ \pm}$hydrogenic states were calculated for different structure parameters (well depth and width) and different fields applied. The calculated $1 s-2 p_{ \pm}$transition energies agree well with experimental data.
\end{abstract}

PACS numbers: 73.20.Dx, 73.20.Hb

An application of the static external electromagnetic fields to doped heterostructures changes the structure of the donor levels in a rather wide range of energy. The application of the magnetic field is of crucial importance in experiments on donors because it lifts the degeneracy of donor states; therefore, the transition energies can be easily measured [1]. There is a number of theoretical papers dealing with the magnetic-field effect on doped heterostructures, e.g., [2-4]. The theoretical study of the influence of the electric field alone on the donor levels in quantum wells $(\mathrm{QW})$ has been presented in the papers $[5,6]$. Yoo et al. [7] have performed a far-infrared magnetoabsorption experiment to investigate the influence of both the electric and magnetic fields on the donor states in the GaAs/ $\mathrm{Ga}_{1-x} \mathrm{Al}_{x} \mathrm{As} \mathrm{QW}$ structure. A theoretical description of the experimental results [7] has been proposed by Latge et al. [8], who applied the single exponential-type trial function. However, the comparison with the experiment [7] does not look satisfactorily, especially for large magnetic fields. In the present paper we have proposed the trial wave function in the form of linear combination of Gaussians. For comparison, we have also performed the calculations using the single exponential-type trial wave function.

We consider a hydrogenic model of a donor impurity located in a quantum well of depth $V_{\mathrm{B}}$ and thickness $L$. The electric and magnetic fields are applied along the growth axis ( $z$ axis). The donor ionization by the electric field is neglected. In the donor units, i.e. the donor Bohr radius as a unit of length and the donor 
rydberg as a unit of energy, the Hamiltonian of our system in the envelope-function approximation can be written as

$$
H=-\nabla^{2}-\frac{2}{r}+V(z)+\frac{1}{4} \gamma^{2} \rho^{2}+\gamma L_{z}+F_{z},
$$

where $F$ is the strength of the electric field, the dimensionless parameter $\gamma=$ $e \hbar B / 2 m c R_{\mathrm{D}}$, the electron-donor distance $r=\sqrt{\rho^{2}+z^{2}}, \rho^{2}=x^{2}+y^{2}, z_{0}$ is the $z$-coordinate of the donor center, $L_{z}=-\mathrm{i} \partial / \partial \theta$ and the potential of the $\mathrm{QW}$ is given by

$$
V(z)=\left\{\begin{aligned}
0, & \text { for }|z|<L / 2, \\
V_{\mathbf{B}}, & \text { for }|z|<L / 2 .
\end{aligned}\right.
$$

We apply the variational procedure in order to find the energy eigenvalues of Hamiltonian (1) and introduce the cylindrical coordinates. For this purpose the two following trial wave functions are used: the single exponential-type function in the form

$$
H_{I}=f(z) \exp (\mathrm{im} \theta) \rho^{|m|} \exp (-\alpha r) \exp (-\beta z)
$$

with the variational parameters $\alpha$ and $\beta$, and the linear combination of Gaussian functions in the form

$$
\psi_{\mathrm{II}}=\sum_{i=1}^{N} c_{i} \varphi_{i}
$$

where

$$
\varphi_{i}=f(z) \exp (\mathrm{i} m \theta) \rho^{|m|} \exp \left(-\alpha_{i} \rho^{2}\right) \exp \left[-\beta_{i}(z+\xi)^{2}\right]
$$

with the variational parameters $\alpha_{i}, \beta_{i}, c_{i}, \xi$. Here, $m$ is magnetic quantum number, $f(z)$ is the wave function of the electron in the potential well without the donor center. We take as $f(z)$ the exact solution in $(\dot{3})$ and the variational wave function in the Gaussian form in (5). When performing the calculations with the trial wave function $\psi_{\mathrm{II}}$, we have taken on $N=5$ terms. The energy eigenvalues are obtained from the minimization of the expectation values of Hamiltonian (1) over the variational parameters.

We have calculated the lowest-lying donor energy levels as functions of the potential-well parameters (well width and depth), the donor center position and the external fields $F$ and $B$. In order to compare the results with the experiment [7], we have evaluated the $1 s-2 p_{+}$transition energies. The $\mathrm{QW}$ parameters are taken on as those used in the paper [7], i.e. $V_{B}=224 \mathrm{meV}$ and $L=500 \AA$. The results are shown in Fig. 1 and Fig. 2. The curves in Fig. 1 have been calculated with the help of trial wave function (3) for the fixed magnetic field $(B=7 \mathrm{~T})$. To account for the distribution of the donors in the experiment [7] over the center of the $\mathrm{QW}$, the three donor center positions in the $\mathrm{QW}$ have been considered, namely $z_{0}=-L / 6$, $0, L / 6$. It can be seen that the experimental points lie within the theoretical curves for extreme donor positions. It can be also noticed that for the donor in the central position, we obtain the best agreement with the experimental results. Therefore, the more extensive calculations with the use of the trial wave function $\psi_{\text {II }}$ have been done for the central donor position. The calculated transition energies are shown in Fig. 2. The results obtained with the help of wave function (3) are also plotted for comparison. It can be seen that we have achieved an improvement of the transition-energy estimates in the whole range of the applied electric and 
magnetic fields. This improvement is especially significant for small values of the electric field.

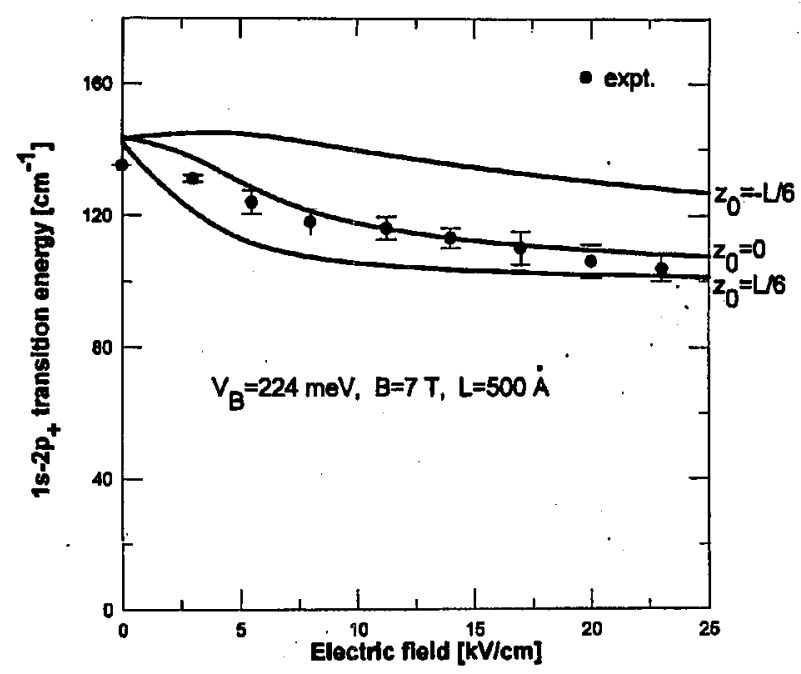

Fig. 1. Calculated $1 s-2 p_{+}$transition energy for the donor in the quantum well as a function of the electric field for the fixed value of the magnetic field $(B=7 \mathrm{~T})$. The results are obtained with the help of trial wave function (3) for the three donor center positions $z_{0}=-L / 6,0, L / 6$.

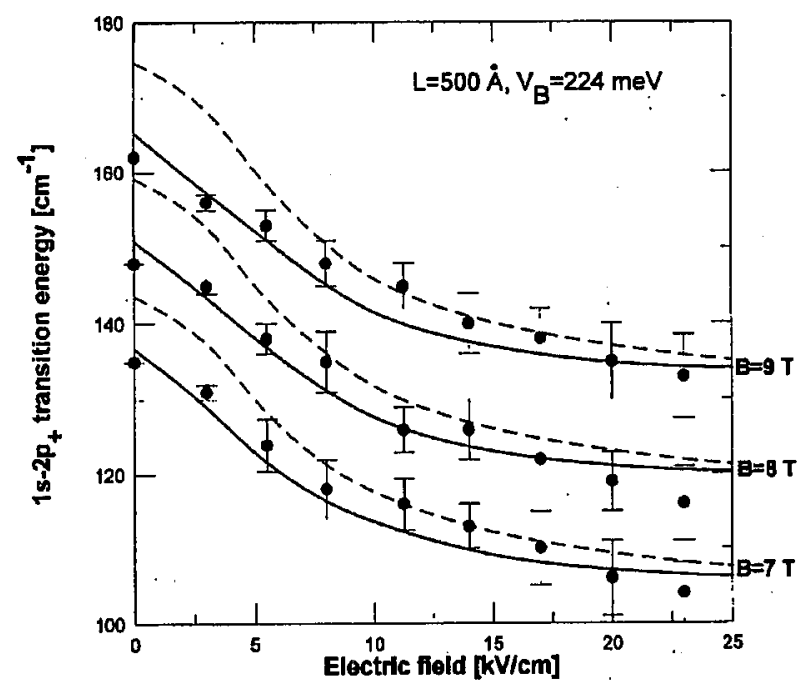

Fig. 2. Calculated $1 s-2 p_{+}$transition energies for the donor in the quantum well as functions of the electric and magnetic fields. Solid (dashed) curves show the results obtained with the help of trial wave function $\psi_{\mathrm{II}}\left(\psi_{\mathrm{I}}\right)$ for the on-center donor position. 
The comparison of the results of our calculations with the experimental data shows that the application of trial function $\psi_{\text {II }}$ (Eqs. (4) and (5)) leads to the best agreement with experiment in the entire range of the electric and magnetic fields applied. The exponential-type trial function $\psi_{\mathrm{I}}$ (Eq. (3)) properly accounts for the properties of the donor in QW excluding the case of the low electric field and strong magnetic field. The estimate obtained with the help of $\psi_{\mathrm{I}}$ becomes more accurate as the magnetic field decreases. We can see that the donor transition energy in QW can be considerably changed by applying the static external electromagnetic fields. In the experiment [7], it corresponds to the range from 12 to $18 \mathrm{meV}$. The $1 s-2 p_{+}$ transition energy is an increasing function of the magnetic field and a decreasing function of the electric field. The present theoretical model correctly reproduces these properties without any fitting parameters.

\section{References}

[1] N.C. Jarosik, B.D. McCombe, B.V. Shanabrook, J. Comas, J. Raltson, G. Wicks, Phys. Rev. Lett. 54, 1283 (1985).

[2] R.L. Greene, K.K. Bajaj, Phys. Rev. B 31, 913 (1985).

[3] J.L. Dunn, E. Pearl, J. Phys., Condens. Matter 3, 8605 (1991).

[4] R. Chen, J.P. Cheng, D.L. Lun, B.D. McCombe, T.F. George, J. Phys., Condens. Matter 7, 3577 (1995).

[5] S. Rolnik, J. Adamowski, Ukr. J. Phys. 40, 680 (1995).

[6] R.B. Santiago, L.E. Oliveira, J.E. Castro, Phys. Status Solidi B 169, K25 (1992).

[7] B. Yoo, B.D. McCombe, W. Schaff, Phys. Rev. B 44, 13152 (1991).

[8] A. Latge, N. Porras-Montenegro, L.E. Oliveira, Phys. Rev. B 51, 2259 (1995). 